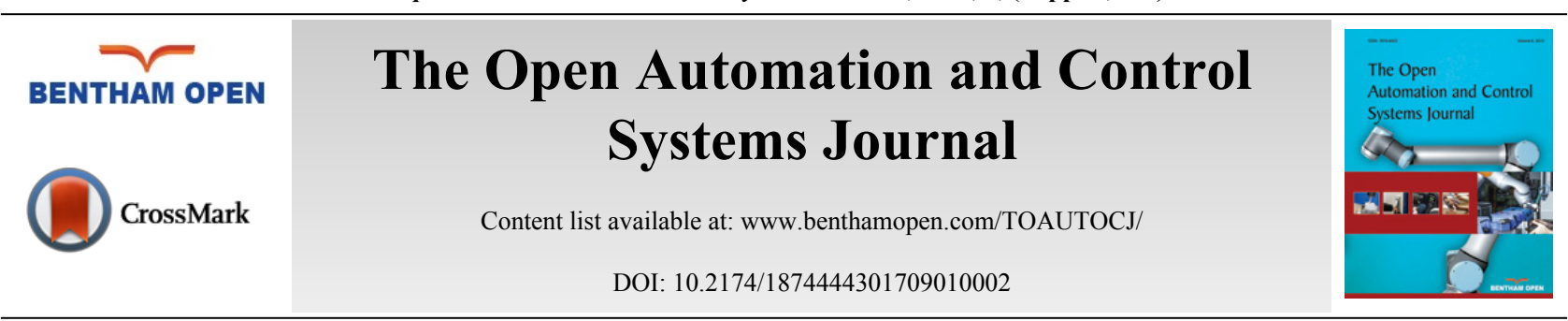

RESEARCH ARTICLE

\title{
Adaptation of Memetic Algorithm with Population Management for the Improvement of the Performances of Flexible Manufacturing Systems
}

\author{
Habiba Houari*, Yamina Houbad, Mehdi Souier, Zaki Sari and Keddari Nassima \\ Manufacturing Engineering Laboratory of Tlemcen (MELT), Abou Bakr Belkaïd University of Tlemcen, PoBox 230, \\ Tlemcen 13000, Algeria
}

Received: October 30, 2016

Revised: February 15, 2017

Accepted: March 30, 2017

\begin{abstract}
:
Objective:

Solving optimization problems is a key point in the constant improving of productivity in the industries.
\end{abstract}

\section{Introduction:}

When traditional methods fail, it is natural to look towards some approximate resolution methods. Memetic algorithm with population management is a Metaheuristic that has been conceived in the last years, and proved its power in the resolution of the difficult optimization problems.

\section{Material and Methods:}

In this paper, our interest is focused on the adaptation of an optimization algorithm called memetic Algorithm with Population Management based on the strategy of management of population to avoid slow or premature convergence and to carry out the excellent executions to solve real time alternative routings selection problem in a Flexible Manufacturing System (FMS), that consists of seven machining centres, a loading and an unloading area, and six different part types which have alternative routings.

\section{Results:}

Simulation results based on two performance indicators which are the production rate, cycle time, work in process and machines utilization rates show that the proposed algorithm performs the best compared to the genetic algorithm.

\section{Conclusion:}

Then we will make a comparison between this algorithm and the genetic algorithm previously used to solve the same problem to get an idea on the most efficient methods for this problem and choose the most effective.

Keywords: Flexible Manufacturing System (FMS), Metaheuristic, Simulation, Memetic algorithm with population management, Optimization problems, The genetic algorithm.

\section{INTRODUCTION}

The evolving manufacturing environment is characterized by a drive toward increasing flexibility. Such as the flexible manufacturing systems (FMS) which provide various advantages like the increase of resources utilization, increase of the productivity, the reduction of work in process ...etc. FMS is a highly automated machine cell consisting

* Address correspondence to this author at Manufacturing Engineering Laboratory of Tlemcen (MELT), Abou Bakr Belkaïd University of Tlemcen, PoBox 230, Tlemcen 13000, Algeria; Tel: 0553061370; E-mail: houari_habiba@yahoo.fr 
of a group of processing work stations, each machine, equipped with a tool magazine, can perform a variety of operations, Interconnected by an integrated transportation system [1].

The system should be flexible, productive and should be able to meet the demands within time bounds at a reasonable cost. An FMS can be considered flexible, if it is able to process parts when they arrive into the system to changing the world as flexibility is the way to stay ahead in business [2].

To take full advantage of the FMS for production, a good scheduling system is needed. Scheduling determines what is going to be made, when, where and with what resources. A desirable scheduling method must include two characteristics easy formulation of the problem and quick identification of semi optimal solutions [3]

Several resolution approaches have been proposed to solve the FMS scheduling problems but there is not an effective solution for all problems due primarily to the complexity of FMS scheduling [4]. Scheduling problems can be considered as more complex in FMS than in traditional manufacturing systems. Scheduling problems can be considered as more complex in FMS than in traditional manufacturing systems. The reason is that not only determining job sequence but also determining which job will be operated at which machine is also crucial for FMS scheduling problem. The FMS scheduling is among the hardest and interesting combinational optimization problem. For this raison, researches used different approaches to solve this problem such as mathematical programming, simulation, and heuristic algorithms... [5]. The choice of the resolution method of the optimization problems depends strongly on the size and complexity on the problem. If the problem is of small size and reduced complexity, an exact method can suffice and lead for an optimal solution. For complex problems, the approached methods, in particular the metaheuristic ones present an interesting alternative.

Many papers show that metaheuristics can obtain good solutions and require less computational time than the exact method. The FMS scheduling is among the hardest and interesting combinational optimization problem. For this reason, researches used different approaches to solve this problem. In the last decades, metaheuristics algorithms were very popular in solving these problems and they did provide many good results such as: Tabu search, simulated annealing, ant colony system, neural network algorithm, genetic algorithm (GA) etc.

Many researchers emphasize that the quality of a metaheuristic optimization approach is largely a result of the effect between intensification and diversification strategies, a good metaheuristic is the one which can control the balance well between these two factors in order to avoid local optima, and a too long exploration [6]. Evolutionary algorithms (EA), particularly the Genetic Algorithms since their development by Holland [7], have been found to provide good approximations of the optimal solution. These algorithms are based on crossover and mutation operators with their probabilities to produce a set of offspring chromosomes. Recently, many papers used hybrid GA to solve optimum problem. Because of their simplicity, and their facility of implementation, they were used for the resolution of several NP-Hard optimization problems, in particular those of scheduling, many variants of Genetic Algorithms have been proposed to improve their performance. Memetic algorithm is the most successful variant in combinatorial optimization [8], also known as hybrid genetic algorithms or genetic local search. These algorithms apply local search procedure to ensure the high quality of individual solutions in the population, having a goal to compensate for the weakness of the genetic algorithm, while allowing a good intensification in the most promising zones of the space of research.

Another variant is the Memetic Algorithm with Population Management (MA/PM) which differs from GA in several respects, the most important ones is the use of a small population of high-quality individuals and the use of population management strategies to control the diversity [6]. Population management strategies can be developed to avoid slow or premature convergence and achieve excellent performance. One possible way of controlling this balance between diversification and intensification strategies is to apply population management, such as in the memetic algorithm with population management approach (MA/PM) [9]. The basic idea of MA/PM is only to allow solution that sufficiently contributes to the diversity of the population and makes ample use of distance measures to be integrated in the population. The distance measure to use depends on the representation of a solution, is much used in scheduling. Needs to be repharese

In this paper we present the adaptation of a MA/PM to solve an alternative routings selection problem in an FMS. The paper is organized as follows: Section 2 describes a literature review on routings selection techniques. Section 3 presents the model of the studied FMS; the MA/PM adaptation for the routing problem is shown in section 4, while Section 5 is devoted to numerical results and computational evaluations. Conclusion is presented in Section 6. 


\section{LITERATURE}

The manufacturing companies are forced to implement systems that can provide flexibility and efficiency because there is an increasing trend towards higher product variety, smaller lot sizes and shorter lead times in the market place. Because of the flexibility of each machine's capability, each operation of a part may be processed on several machines and its processing time may vary a little on these different machines. Also, a part can be finished processing through one of several different operation sequences. That means an alternative operation routing is allowed [1]. Routing flexibility can be regarded as the main contributor to the flexibility of an FMS. It is the capability of processing a part through varying routes by using alternative machines to produce a set of parts economically and efficiently [10]. Enhancing the performance of an FMS using routing flexibility in scheduling is the motivating factor for this paper. A vast amount of literature concerning alternate routings and dynamic scheduling is available [11]. Grinsted [12] discussed the benefits of using alternative routes in a job shop and suggested four decision rules that may be useful. Singh and Mohanty [13] proposed a fuzzy multiobjective approach for solving the routing problem in the job shop environment. Chang et al. [14] used a simulation to study a system with eight machines and eight part types. They found that routing flexibility improved system performance, especially when combined with a look-ahead dispatching technique that sent the part to the machine with the least load. environment Warren Liao [15] proposed a linear model to program a whole numbers to determine the optimal routing which minimizes the production cost by taking account of the volume of production and the capacity of the machines. Rahul Capih and Subhash Wadwa [16] studied the effect of some key design and control parameters on the performance of a hypothetical FMS; they presented a framework based on a Taguchi experimental design for studying the nature of the impact of varying levels of routing flexibility on the performance of an FMS. . Ro and Kim [17] used multiple criteria decision-making techniques to test alternate dispatching rules in an FMS with routing flexibility In the existing literature on dynamic part routing, Caprihan and Wadhwa [18] showed the effect of the variation of the levels of routing flexibility (the number of alternative routings taken by a type of part) on the performances of an FMS judged by the makespan. Caprihan and Kumar [19] proposed a strategy of scheduling taking into account the flexibility of routings based on fuzzy logic in an FMS. Chunwei and Zhiing $\mathrm{Wu}$ [20] suggested a genetic algorithm (GA) to solve the job-sequencing problem for a production shop that is characterized by flexible routing and flexible machines. They attempted to deal with situations similar to an actual FMS environment, included the requirements of routing selection, machine selection, operations sequence selection, and dynamic scheduling. Chen and Chung [21] proposed three alternative loading models aimed at maximizing the operation assignments and consequently the inherent FMS flexibility. The results demonstrated the importance of utilizing an alternate routing technique in the FMS

Ümit Bilge et al. [22] presented a study which relates to the flexibility of routing. This study introduced three new approaches, including a fuzzy logic approach, for dynamic part routing. An-Yuan Chang [23] presented a research to measure the flexibility of routing; they proposed, inter alia, of the mathematical models in order to evaluate the effectiveness of the routings, the versatility thus as the variety of these routings. Rajamani D et al. [24] proposed three models of programming of whole numbers to study the effect of alternative routings of the parts while taking account of the use of the resources whose objective is to minimize the investment. Andrea Rossi and Gino Dini [25] proposed an ant colony optimization-based software system for solving FMS scheduling in a job-shop environment with routing flexibility, sequence-dependent setup and transportation time. Min-Chun Yu and Timothy J. Greene [11] presented a background and a rational for a routing flexibility measure for a multi-stage flow shop, an operational measure of routing flexibility in a multi-stages multi-product production system. Garavelli [26] conducted a simulation study on the performance of several flexible manufacturing systems, each of which was characterized by a specific degree of routing flexibility. He found that instead of complete flexibility, a system with limited flexibility performed better in terms of lead time and work-in-process. ElMekkawy and ElMaraghy [27] utilized flexible routing in order to avoid system deadlock caused by machine breakdowns and downtimes. Shukla and Chen [28] proposed a decision support system framework to assist in the control of an FMS through intelligent part launching. The proposed system makes use of a heuristic based on the pull concept and a neural network model. Haq et al. [29] proposed an enumerative heuristic algorithm for scheduling an FMS where in the production schedule is integrated with the MHS schedule. Joseph and Sridharan [4] studied effects of routing flexibility, sequencing flexibility and scheduling decision rules on the performance of a flexible manufacturing system.

We can find also the work of Mahmoodi and Mosier [30] in which he studied the effect of priority rules and levels of routing flexibility on the different performances of the FMS. We find also the work of Saygin and Kilick Who proposed a platform that integrates the flexible planning and predictive scheduling; they presented a concept named 
Dissimilarity Maximization Method (DMM) to minimize congestion in an FMS. The idea in this method is to maximize dissimilarities between the occupied routings; in their algorithm each routing contains only one part at the same time. The efficiency of that rule in solving routings selection problems has been proven in [31] and Ghomri [32] where it outperformed the other two routings selection rules FIFO/FA (First-In First-Out/ First Available) and EPL (Equal Probability Loading) if each machine uses the FIFO as sequencing rule. In [29] the authors studied the DMM rule and proposed modified DMM rule which is a modification of the DMM rule which aimed to maintain the same idea that is the maximization of the dissimilarity coefficients for selection of different alternative routings but by affecting several parts to a single routing.

We can find also in [2] the adaptation of several metaheuristics (Ant colony, genetic algorithm, simulated annealing, taboo search, particle swarms, and electromagnetism) for solving routings selection problem in an FMS with an important number of parts but in delayed time, thereafter they have successfully applied these metaheuristics on the same problem in real time.

\section{RESENTATION OF FLEXIBLE MANUFACTURING SYSTEM ENVIRONMENT}

The FMS model studied is drawn from the literature (Saygin and Kilick, 1999; Hassam and Sari, 2010 [33]). It is composed from seven machines and two stations, one is a loading station and the other is an unloading one. The system will process six kinds of parts. Each machine has an input and an output queue. The loading station also has an input queue. Fig. (1) represents the model's configuration, and Table 1 shows possible alternative routings for each parts kind with the processing time for each machine.

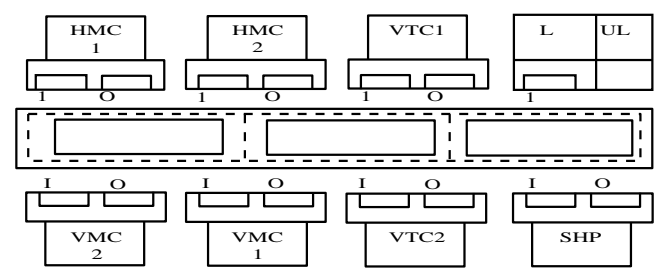

Fig. (1). Model FMS configuration. I: input buffer, O: output buffer.

Table 1. Alternative routings and parts processing times.

\begin{tabular}{|c|c|c|c|}
\hline Parts Kinds & Arrival Rate & Routing Number & Routings and Processing Time(min) \\
\hline \multirow[t]{4}{*}{ A } & \multirow[t]{4}{*}{$17 \%$} & 1 & L- VTC1(30)- VMC1(20)- UL \\
\hline & & 2 & L- VTC1(30)- VMC2(20)- UL \\
\hline & & 3 & L- VTC2(30)- VMC1(20)- UL \\
\hline & & 4 & L- VTC2(30)- VMC2(20)- UL \\
\hline \multirow[t]{4}{*}{ B } & \multirow[t]{4}{*}{$17 \%$} & 5 & L- VTC1(20)- VTC2(1)- VMC1(15)- UL \\
\hline & & 6 & L- VTC1(20)- VTC2(1)- VMC2(15)- UL \\
\hline & & 7 & L- VTC2(20)- VTC2(1)- VMC1(15)- UL \\
\hline & & 8 & L- VTC2(20)-SHP(1)- VMC2(15)- UL \\
\hline \multirow[t]{4}{*}{$\mathrm{C}$} & \multirow[t]{4}{*}{$17 \%$} & 9 & L- VTC1(40)-SHP(1)- VMC1(25)- UL \\
\hline & & 10 & L- VTC1(40)-SHP(1)- VMC2(25)- UL \\
\hline & & 11 & L- VTC2(40)-SHP(1)- VMC1(25)- UL \\
\hline & & 12 & L- VTC2(40)-SHP(1)- VMC2(25)- UL \\
\hline \multirow[t]{8}{*}{$\mathrm{D}$} & \multirow[t]{8}{*}{$21 \%$} & 13 & L- VTC1(40)-SHP(1)- VTC1(20)- HMC1(35)- UL \\
\hline & & 14 & L- VTC1(40)-SHP(1)- VTC120)- HMC2(35)- UL \\
\hline & & 15 & L- VTC1(40)-SHP(1)- VTC2(20)- HMC1(35)- UL \\
\hline & & 16 & L- VTC1(40)-SHP(1)- VTC2(20)- HMC2(35)- UL \\
\hline & & 17 & L- VTC2(40)-SHP(1)- VTC1(20)- HMC1(35)- UL \\
\hline & & 18 & L- VTC2(40)-SHP(1)- VTC1(20)- HMC2(35)- UL \\
\hline & & 19 & L- VTC2(40)-SHP(1)- VTC2(20)- HMC1(35)- UL \\
\hline & & 20 & L- VTC2(40)-SHP(1)- VTC2(20)- HMC2(35)- UL \\
\hline
\end{tabular}


(Table 1) contd....

\begin{tabular}{|c|c|c|c|}
\hline Parts Kinds & Arrival Rate & Routing Number & Routings and Processing Time(min) \\
\hline \multirow[t]{8}{*}{$\mathrm{E}$} & \multirow[t]{8}{*}{$20 \%$} & 21 & L- VTC1(25)-SHP(1)- VTC1(35)- HMC1(50)- UL \\
\hline & & 22 & L- VTC1(25)-SHP(1)- VTC1(35)- HMC2(50)- UL \\
\hline & & 23 & L- VTC1(25)-SHP(1)- VTC2(35)- HMC1(50)- UL \\
\hline & & 24 & L- VTC1(25)-SHP(1)- VTC2(35)- HMC2(50)- SD \\
\hline & & 25 & L- VTC2(25)-SHP(1)- VTC1(35)- HMC1(50)- UL \\
\hline & & 26 & L- VTC2(25)-SHP(1)- VTC1(35)- HMC2(50) UL \\
\hline & & 27 & L- VTC2(25)-SHP(1)- VTC2(35)- HMC1(50)- UL \\
\hline & & 28 & L- VTC2(25)-SHP(1)- VTC2(35)- HMC2(50)- UL \\
\hline \multirow[t]{2}{*}{$\mathrm{F}$} & \multirow[t]{2}{*}{$8 \%$} & 29 & L-FH1(40)- UL \\
\hline & & 30 & L-FH2(40)- UL \\
\hline
\end{tabular}

- L: Loaded Station

- UL: Unloaded Station

- VTC1, VTC2: Vertical Turning Centers

- VMC1, VMC2: Vertical Milling Machines

- HMC1, HMC2: Horizontal Milling Machines

Operations in our FMs are based on the following assumptions:

- The alternative routings is known for each kind of parts before the beginning of the production.

- The processing time is determinate and it includes the time spent changing tools and the execution time of the machine.

- Each machine can process one part at a time.

\section{THE MA|PM ADAPTATION FOR THE ALTERNATIVE ROUTING SELECTION PROBLEM}

This section gives a description of the designed adaptive memetic algorithm with population management (MAPM) to solve the studied routing selection problem. The basic principle of the genetic algorithms is the imitation the natural process of reproduction. It begins with a population of strings created randomly. Thereafter, each string in the population is evaluated. The population is then operated by three main operators; reproduction, crossover and mutation to create a better population. In spite of the great success, generally, met by the genetic algorithms in resolution of many NP-Hard optimization problems, they can have certain weaknesses.

Several improvements were introduced with the genetic algorithms, and several variants are proposed to make these techniques more powerful, among which hybrid algorithms of Moscato [8] introducing a local research, and giving birth to the algorithms memetic.

A more advanced form of the genetic approach is that proposed by Sorensen and Sevaux [6]. In which a measurement of distance is used, in order to bring certain diversity in the population. The proposed algorithm uses the population management, which controls the diversity of population of high-quality solutions. The algorithm proposed bears the name of algorithm memetic with population management (MA|PM).

Both memetic algorithms with population management and Genetic algorithm are evolutionary method and the main features are population-based. In contrast to genetic algorithms, memetic algorithms with population management is founded on the premise that systematic designs and methods for creating new solutions afford significant benefits beyond those derived from resource to randomization. It uses strategies for search diversification and intensification that have proved effective in a variety of optimization problems. Many researchers agree that the quality of a metaheuristic optimization approach is largely a result of the interplay between intensification and diversification strategies.

The basic idea of MA|PM comes from the management of the population; thanks to a measurement of distance in the space of the solutions. This operation entitle to control the diversity of the individuals by filtering the entry of the children in the new population and thus preventing the stagnation and premature convergence. In contrast to traditional memetic algorithms, the activation of local searcher does not depend on the diversity parameters in solutions space.

The MA|PM and GA can also be compared according to intensification and diversification as in Table $\mathbf{2}$ : 
Table 2. compare between the MA|PM and GA.

\begin{tabular}{|c|c|c|}
\hline Metahuristic Methods & Intensification & Diversification \\
\hline MA|PM: & Selection \\
-Small population & The local search \\
-High-quality & Update of $\Delta$ & \\
-Local improvement & & Mutation \\
GA: & Selection \\
-Large population & Crossover & \\
-Mixed-quality solutions & Replacement & \\
-No local improvement & & \\
\hline
\end{tabular}

The main components of the proposed algorithm are as follow and significance of each parameter in our adaptation.

\subsection{The Coding of the Solutions}

As in a genetic algorithm, a coding is a representation of a solution in the form of chromosomes, named individual. In our case, an individual is a chain containing the selected routings of the parts present in the infinite file. Fig. (2) gives an explanatory example, for a capacity of queue equalizes to 4. Let us suppose that the first four parts contained in the infinite file are as follows: C, A, F and B. the routings corresponding to these parts are: 9, 10, 11, and 12 for C, 1, 2, 3 and 4 for A, 29 and 30 for F, and 5, 6, 7 and 8 for B. coding is thus a chain which comprises one of the possible routings for each part.

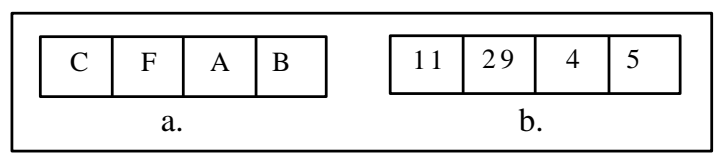

Fig. (2). a. A set of parts; b. A possible solution corresponding to the considered set of parts.

\subsection{The Selection Operator}

The proportional selection has to choose two individuals parents $\mathrm{p} 1$ and $\mathrm{p} 2$, which will be submitted to the operator of crossing to train two new individuals, known as children: $\mathrm{c} 1$ and $\mathrm{c} 2$.

\subsection{The Crossover Operator}

Crossover has to choose a place of cut along the two individuals 'parents' $\mathrm{p} 1$ and $\mathrm{p} 2$, and then to connect the right part of the one to the left part of the other, and conversely Fig. (3) presents an example of change:

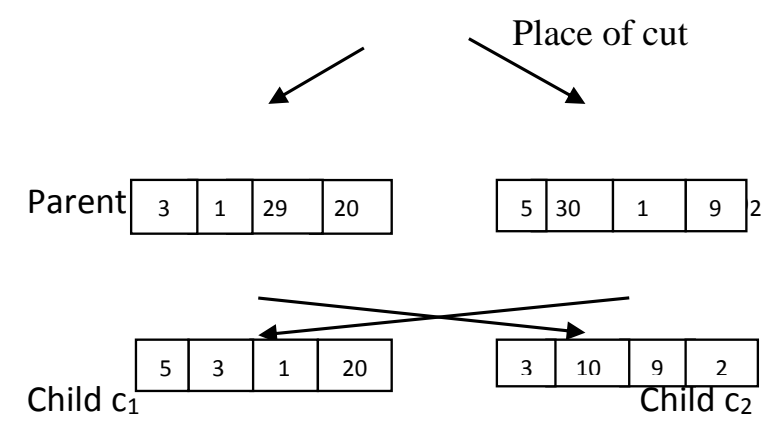

Fig. (3). The crossover operator.

\subsection{The Mutation Operator}

Mutation is a simple operator consisting of random changes in the value of genes in a chromosome. If recombination is supposed to exploit the current solutions to find better ones, mutation is supposed to help for the exploration of the whole search space.

The mutation example has to change the routings of certain parts randomly, according to their types is given in (Fig. 4). 


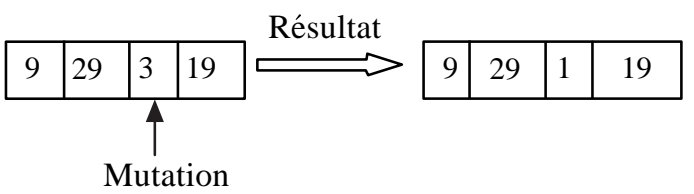

Fig. (4). The mutation operator.

\subsection{The Parameter of Diversity $\Delta$}

MA|PM uses the population management in order to controls the diversity of population of high-quality solutions. For this purpose a distance measured that determines for each pair of solutions their relativedistance (or similarity) in the solution space is required, the distance of a given solution $\mathrm{c}$ to the population $\mathrm{P}$ can becalculated as follows:

$$
d_{p}(c)=\min _{s \in p} d(c, p)>\Delta
$$

The solution that has a small distance to another solution already in the population, will not contribute much to the diversity of a population. Therefore, a solution is not added to the population if its distance to the population is below acertain threshold $\Delta[5]$.

The parameter $\Delta$ is the important parameter in MA|PM, so it makes possible to have, intelligently, a good diversification in the algorithm, which balance the good intensification obtained by the local research used in an algorithm memetic. The parameter of diversity is often measured in the following way:

$$
\left\{\Delta=\sum \Delta\left(S_{i}, S_{j}\right)\right\}
$$

The distance is the number of characteristic different between two solutions. if $\mathrm{S} 1=(9,30,10,5)$ and $\mathrm{S} 2=(20,15,10,2)$ then $\Delta(\mathrm{S} 1, \mathrm{~S} 2)=3$. More two individuals are distant they are regarded as being different.

\subsection{The Objective Function}

All metaheuristics tempt to find a solution aiming to minimize or maximize (according to the problem) a function known as 'objective function'. Our aim is to find the best routing for each part as soon as it arrives to the loading station. To find the fitness of chromosome $\mathrm{S}$ in our work, the objective function is the load produces routings, mathematicians have proven that the higher the values are very close over their product is great (geometric mean).

For this reason, we take into account, at each iteration, criteria concerning the system state like the production rate. Thus, using the studied metaheuristic, we try to balance the routings loads not in term of parts number but in term of processing time. To implement this concept, the routing loads product should be maximized. For that, we took into account the $\mathrm{n}$ first parts in the infinite queue (or the order list), where $\mathrm{n}$ equals the loading station size. The infinite queue contains parts waiting to pass into the loading station. Routings of these parts are modified, at each iteration, according to the MA|PM algorithm principle. For each routing we calculate its load in order to maximize the product of these routings loads.

The maximization of this function (the product of the operating time is defined by this function:

$$
F(t)=\prod_{j=1}^{30}\left(\sum_{i=1}^{m} O(i, j)\right)
$$

$m$ : the number of machines

$j:$ the number of routings

$O$ : operating times

\subsection{The Local Search Procedure}

The local search procedure used in the memetic algorithm with population management permits the improvement of obtained solutions. In our case, the local search procedure is the simple descent, given in the following way:

1. For each solution $x$

2. Repeat

3. Find a solution $x^{\prime}$ in the neighborhood of $x$ 
4. If $\mathrm{f}\left(\mathrm{x}^{\prime}\right)<\mathrm{f}(\mathrm{x})$ then

5. $\mathrm{x}^{\prime} \leftarrow \mathrm{x}$

6. End if

7. Until stopping criterion is satisfied

In our adaptation, a neighbourhood solution of $\mathrm{x}$ is found by modifying some parts routings in $\mathrm{x}$ according to their types, this modification is done randomly, and the stopping criterion is the number of iterations.

The memetic adaptation of the algorithm with population management is as follows:

1. If there is a free place in the loading station then

2. Generate an initial population $P$ of solutions, by affecting $N$ first parts of the infinite file to routings randomly according the parts type.

3. As long as the criterion of stop is not reached make

For each individual

Evaluate the objective function

If there is improvement of the objective function then accept the improveing solution End for

4. Fix the parameter of diversity $\Delta$

5. Repeat

6. Select two parents' $\mathrm{p} 1$ and $\mathrm{p} 2$

7. Apply the operator of crossing to $\mathrm{p} 1$ and $\mathrm{p} 2$ (one obtains two children $\mathrm{c} 1$ and $\mathrm{c} 2$ )

8. Apply the operator of local research to $\mathrm{c} 1$ and $\mathrm{c} 2$

9. For any resulting child $\mathrm{C}$

As long as $\mathrm{C}$ satisfies the condition of addition

Mutation of C

End as long as

Delete solution of population

Addition of solution $\mathrm{p} \leftarrow \mathrm{p} \mathrm{U} \mathrm{C}$

End for

10. Update the parameter of diversity $\Delta$

11. Until satisfying the criterion of stop

12. End as long as

13. End if

\section{RESULTS AND DISCUSSIONS}

In this section a number of experiments are carried out which outlines the effectiveness of both the algorithms described above. The purpose of these experiments is to compare the performance of algorithm memetic with population management approach adapted to the genetic algorithm approach adapted by [2] for solving the same alternative routings selection problem.

The proposed algorithm is coded in Java and simulated over 2000 hours with a warm up time of 3000 hours and ten replications are made. The study is realized in two parts: with and without presence of breakdown in the system. A breakdown occurs according to an exponential distribution with an MTBF=100 hours and MTTR=2 hours.

In all the remainder of the paper, the queues capacities is fixed at 2 , therefore the parameter of diversification $\Delta$ takes one of two values 0 or 1 .

\subsection{The Choice of the Parameter of Diversity $\Delta$}

The performance of metaheuristic depends essentially on the choice of its parameters. In MA|PM, the parameter of diversity is the important parameter in the algorithm. The value of the parameter $\Delta$ depends mainly on the dimension of the chromosome, because it measures the dissimilitude between two individuals (chromosomes).

In our case, the size of a chromosome represents the capacity of the queue, and therefore the maximum value that $\Delta$ can take is equal to the capacity of queue. 
Table 3 presents the different performances criteria according to the diversity parameter $\Delta$, for a queues capacity equals to 2 et 6 , and an arrival rate of the parts equals to $1 / 19$ and $1 / 18$. For a $\Delta$ equals to 0 , the algorithm behaves like a traditional algorithm memetic, but the results remain acceptable, while for $\Delta=1$, production rate increases, and cycle time decreases.

Table 3. The different criteria of performances according to the parameter of diversity $\Delta$,for a capacity of queue equals to 2 et 6 , and anarrive rate of the part equals to $1 / 18$.

\begin{tabular}{|c|c|c|c|}
\hline Capacity of Queue & Parameter $\Delta$ & Production Rate (\%) & Cycle Time (min) \\
\hline \multirow{2}{*}{2} & $\Delta=0$ & 90,04 & 176,07 \\
\cline { 2 - 4 } & $\Delta=1$ & 92,41 & 335,67 \\
\hline \multirow{4}{*}{6} & $\Delta=0$ & 87.71 & 342,04 \\
\cline { 2 - 4 } & $\Delta=1$ & 87.86 & 335,62 \\
\cline { 2 - 4 } & $\Delta=2$ & 87.76 & 324,21 \\
\cline { 2 - 4 } & $\Delta=3$ & 87.84 & 327,58 \\
\cline { 2 - 4 } & $\Delta=4$ & 87.94 & 329,73 \\
\hline
\end{tabular}

\subsection{Production Rate}

Table 4 shows that the memetic algorithm with management of population gives the good results compared with the genetic algorithm.

Table 4. Production rate of parts for a queues capacity equals to 2et 6.

\begin{tabular}{|c|c|c|c|c|c|c|}
\hline C.Q & T.A.R(1/min) & $\mathbf{1 / 5}$ & $\mathbf{1 / 1 0}$ & $\mathbf{1 / 1 5}$ & $\mathbf{1 / 2 0}$ & $\mathbf{1 / 2 5}$ \\
\hline \multirow{2}{*}{2} & AG & 23.31 & 47.25 & 71.08 & 98.44 & 99.99 \\
\cline { 2 - 7 } & MA|PM & 24.31 & 48.62 & 72.84 & 99.99 \\
\hline \multirow{2}{*}{6} & AG & 24.17 & 48.26 & 72,40 & 99,99 & 99.99 \\
\cline { 2 - 7 } & MA|PM & 24,39 & 48,86 & 72,5 & 99,99 \\
\hline
\end{tabular}

T.A.R: Arrival rate of parts. C.Q: Capacity of queue. T.M: The types of machines MA|PM: memetic algorithm with management of population. GA: genetic algorithm.

Table 5 gives the evolution of the rate of production for various values of the queues capacity. It is clear that the memetic algorithm with population management gives better results compared with those of the genetic algorithm.

Table 5. Production rate for Arrival rate of parts =1/5,1/20.

\begin{tabular}{|c|c|c|c|c|c|}
\hline \multirow{2}{*}{ T.A.R } & C.Q & $\mathbf{2}$ & $\mathbf{4}$ & $\mathbf{6}$ \\
\hline \multirow{2}{*}{$\mathbf{1} 1 \mathbf{5}$} & AG & 23,70 & 23,93 & 24,17 \\
\cline { 2 - 6 } & MA|PM & 24.39 & 24,34 & 24,39 \\
\hline \multirow{2}{*}{$\mathbf{1} / \mathbf{2 0}$} & AG & 98,44 & 99,99 & 99,24 \\
\cline { 2 - 6 } & MA|PM & 99,99 & 99,99 & 99,51 \\
\hline
\end{tabular}

\subsection{Cycle Time}

Let's now examine the cycle time of the system. (Tables 6 and 7) give the cycle time values for different arrival rate values.

Table 6. Cycle time of parts for a queues capacity $=2$ and 6.

\begin{tabular}{|c|c|c|c|c|c|c|}
\hline \multirow{2}{*}{ C.Q } & $\begin{array}{c}\text { T.A.R } \\
(\mathbf{1} / \mathbf{m i n})\end{array}$ & $\mathbf{1 / 5}$ & $\mathbf{1 / 1 0}$ & $\mathbf{1 / 1 5}$ & $\mathbf{1} / \mathbf{2 5}$ \\
\hline \multirow{2}{*}{$\mathbf{2}$} & AG & 168.10 & 168.90 & 169.80 & 132.70 \\
\cline { 2 - 7 } & MA|PM & 166.72 & 171.60 & 170.80 & 127.40 \\
\hline \multirow{2}{*}{$\mathbf{6}$} & AG & 338,10 & 332,80 & 332,00 & 121,30 & 98.70 \\
\cline { 2 - 7 } & MA|PM & 325.90 & 340.73 & 327.32 & 98,90 \\
\hline
\end{tabular}


Table 7. Cycle time of parts for the rates of creations of parts $=1 / 5,1 / 20$.

\begin{tabular}{|c|c|c|c|c|c|}
\hline $\begin{array}{c}\text { T.A.R } \\
(\mathbf{1} / \mathbf{m i n})\end{array}$ & $\mathbf{C . Q}$ & $\mathbf{2}$ & $\mathbf{4}$ & $\mathbf{6}$ & $\mathbf{8}$ \\
\hline \multirow{2}{*}{$\mathbf{1} / \mathbf{5}$} & AG & 168,10 & 249,90 & 338,10 & 417,00 \\
\cline { 2 - 6 } & MA|PM & 166.07 & 245.02 & 325,90 & 427,66 \\
\hline \multirow{2}{*}{$\mathbf{1 / 2 0}$} & AG & 132,70 & 118,00 & 121,30 & 119,70 \\
\cline { 2 - 6 } & MA|PM & 127,42 & 122,50 & 118,70 & 125,01 \\
\hline
\end{tabular}

Comparing the cycle times of the two algorithms, we found that values obtained by the algorithm memetic with population management are lower than those obtained by the genetic algorithm.

\subsection{Machines Utilization Rate}

Machines utilization rate is an important criterion in performance measurement of production systems. The machines utilization rate was defined by the percentage of the time of use of the machines compared to the time of simulation.

Two cases are taken into account, with and without breakdowns. We present the machines utilization rate of the VTC, VMC, HMC and SHP machines. The breakdowns are introduced on the resources each 100 hours. The breakdown will steel 2 hours following an exponential law.

Tables ( 8 and 9) give Machines utilization rate for different arrival rate values with and without breakdowns.

Table 8. Machines utilization rate for a queues capacity $=\mathbf{2}$.

\begin{tabular}{|c|c|c|c|c|c|c|}
\hline \multirow{2}{*}{ T.M } & $\begin{array}{c}\text { T.A.R } \\
(\mathbf{1} / \mathbf{m i n})\end{array}$ & $\mathbf{1 / 5}$ & $\mathbf{1 / 1 0}$ & $\mathbf{1 / 1 5}$ & $\mathbf{1 / 2 0}$ & $\mathbf{1 / 2 5}$ \\
\hline \multirow{2}{*}{ VTC } & $\mathrm{AG}$ & 90,96 & 90,85 & 90,98 & 92,74 & 75,69 \\
\cline { 2 - 7 } & $\mathrm{MA} \mid \mathrm{PM}$ & 91,82 & 91,81 & 91.87 & 94.08 & 75,69 \\
\hline \multirow{2}{*}{ VMC } & $\mathrm{AG}$ & 28,72 & 28,36 & 28,60 & 31,94 & 25,54 \\
\cline { 2 - 7 } & $\mathrm{MA} \mid \mathrm{PM}$ & 31.18 & 31.26 & 31.02 & 32,50 & 25,52 \\
\hline \multirow{2}{*}{ HMC } & $\mathrm{AG}$ & 42,36 & 42,57 & 42,47 & 40,99 & 33,88 \\
\cline { 2 - 7 } & $\mathrm{MA} \mid \mathrm{PM}$ & 40,76 & 40,88 & 41,13 & 41,45 & 33,92 \\
\hline \multirow{2}{*}{ SHP } & $\mathrm{AG}$ & 2,387 & 2,399 & 2,393 & 2,300 & 1,907 \\
\cline { 2 - 7 } & $\mathrm{MA} \mid \mathrm{PM}$ & 2,305 & 2,301 & 2,315 & 2,333 & 1,910 \\
\hline
\end{tabular}

Table 9. Machines utilization rate with introduction of the breakdowns for a queues capacity=2.

\begin{tabular}{|c|c|c|c|c|c|c|}
\hline \multirow{2}{*}{ T.M } & $\begin{array}{c}\text { T.A.P } \\
(\mathbf{1} / \mathbf{m i n})\end{array}$ & $\mathbf{1 / 5}$ & $\mathbf{1 / 1 0}$ & $\mathbf{1 / 1 5}$ & $\mathbf{1 / 2 0}$ & $\mathbf{1 / 2 5}$ \\
\hline \multirow{2}{*}{ VTC } & $\mathrm{AG}$ & 88,24 & 88,32 & 88,29 & 88,53 & 76,57 \\
\cline { 2 - 7 } & $\mathrm{MA} \mid \mathrm{PM}$ & 88,68 & 88,68 & 88,55 & 88,74 & 76,62 \\
\hline \multirow{2}{*}{ VMC } & $\mathrm{AG}$ & 28,04 & 28,05 & 27,72 & 27,10 & 25,02 \\
\cline { 2 - 7 } & $\mathrm{MA} \mid \mathrm{PM}$ & 30.34 & 30,50 & 30,54 & 30,16 & 25,02 \\
\hline \multirow{2}{*}{ HMC } & $\mathrm{AG}$ & 42,36 & 42,57 & 42,47 & 40,99 & 33,88 \\
\cline { 2 - 7 } & $\mathrm{MA} \mid \mathrm{PM}$ & 41,25 & 41,22 & 41,36 & 41,63 & 34,62 \\
\hline \multirow{2}{*}{ SHP } & $\mathrm{AG}$ & 2,324 & 2,322 & 2,330 & 2,340 & 1,950 \\
\cline { 2 - 7 } & $\mathrm{MA} \mid \mathrm{PM}$ & 2,251 & 2,240 & 2,246 & 2,247 & 1,950 \\
\hline
\end{tabular}

It is clearly seen that the memetic algorithm with population management is powerful than the GA in the T and FV machines utilization rates, but for SHP and HMC machines utilization rate, best results are given by the GA.

The machines utilization rates of creations of parts $=1 / 20,1 / 25$ values for the MA/PM are higher than those obtained by the genetic algorithm.

According to the experimental results, it can be seen that results obtained by the memetic algorithm with population management are, generally, better than those obtained by the Genetic Algorithm, so we can conclude that the parameter $\Delta$ is the most important parameter and the population management play an important role in the MA/PM process.

Also we can say that including a high quality heuristic solution can help the GA to improve its performance by 
reducing the probability of its premature convergence. In addition, the diversity control has a great effect on the metaheuristic performances.

\section{CONCLUSION}

In this paper we have presented a memetic algorithm with population management approach for solving an alternative routings selection problem in an NP-hard combinatorial problem. A memetic algorithm with population management is an extension of the traditional genetic algorithm. It is based on the management of population, by using the distance measurement in the solutions space, to control the diversity of a small population of solutions of highquality.

The goal is the improvement of certain performance criteria of the studied system. MA/PM using a population management strategy to control the diversity of the population and having dynamic parameters and local Searcher which are adaptively employed in order to explore the decision space .

Our objective is to determine the performance of the memetic algorithm with population management compared to the genetic algorithm for a scheduling problem in an FMS. The results (except those of the utilization ratio of machines HMC and SHP) show the performance of the memetic algorithm with population management with and without breakdowns.

\section{CONSENT FOR PUBLICATION}

Not applicable.

\section{CONFLICT OF INTEREST}

The author (editor) declares no conflict of interest, financial or otherwise.

\section{ACKNOWLEDGEMENTS}

Declared none.

\section{REFERENCES}

[1] Z. Chunwel, and W. Zhining, "“A Genetic Algorithm Approach to the Scheduling of FMSs with Multiple Routes”, The Shanghai Jiaotong University International", J. Flexible Manuf. Syst., vol. 13, pp. 71-88, 2001. [http://dx.doi.org/10.1023/A:1008148313360]

[2] M. Souier, A. Hassam, and Z. Sari, "Meta-heuristics for real time routing selection in FMS", In: Lyes Benyoucef and Bernard Grabot (Ed.), Artificial Intelligence Techniques for Networked Manufacturing Enterprises Management: Springer-Verlag, London, 2010 , pp. $221-247$.

[3] Y.W. Kim, "FMS scheduling based on timed Petri Net model and reactive graph search", Appl. Math. Model., vol. 31, no. 6, pp. 955-970, 2007. [http://dx.doi.org/10.1016/j.apm.2006.10.023]

[4] O.A. Joseph, and R. Sridharan, "Effects of routing flexibility: sequencing flexibility and scheduling decision rule on the performance of a flexible manufacturing system", In: Int J Adv Manuf Technol, vol. 56. Springer-Verlag London Limited, 2011, no. 1-4, pp. 291-306. [http://dx.doi.org/10.1007/s00170-011-3158-0]

[5] "Selection of dispatching rules in FMS: ANP model based on BOCR with choquet integral", Int. J. Adv. Manuf. Technol., vol. 49, no. 5-8, pp. 785-801, 2009

[6] M. Sevaux, "Métaheuristiques Stratégie pour l'optimisation de la production de biens et de services", Habilitation à diriger des recherches Laboratoire d'Automatique, de Mécanique d'informatique Industrielles et Humaines du CNRS (UMR CNRS 8530) dans l'équipe systèmes de production, .

[7] J.H. Holland, Adaptation in natural and artificial systems, Michigan Press Univ: Ann Arbor, MI, 1975.

[8] P. Moscato, "On evolution, search, optimization, genetic algorithms and martial arts: Towards memetic algorithms", In: Technical report., Caltech Concurrent Compulation Program, 1989.

[9] B.O. Boukef, Sur l'ordonnancement d'ateliers job-shopflexibles ET flow-shop en industries pharmaceutiques: Optimisation par algorithmes génétiques et essaims particulaires, 2010.

[10] O.A. Joseph, "Evaluation of routing flexibility of a flexible manufacturing system using simulation modelling and analysis", Int. J. Adv. Manuf. Technol., vol. 56, no. 1-4, pp. 273-289, 2011 [http://dx.doi.org/10.1007/s00170-011-3153-5] 
[11] Min-Chun Yu, and Greene J.Timothy, "ShopAn operational measure of routing flexibility in a multi-stage multi-product production system", Int J Adv Manuf Technol, vol. 43, pp. 357-364, 2009. Springer-Verlag London Limited, 2008

[12] S. Grinsted, “Alternative Routing: Some Rules for the "Where-Next" Decision", In: Proceedings of the Second International Conference of European Operations Management Association, Enschede, the Netherlands , pp. 171-177,1995

[13] N. Singh, and B.K. Mohanty, "A Fuzzy Approach to Multi-objective Routing Problem with Applications to Process Planning in Manufacturing Systems", Int. J. Prod. Res., vol. 29, no. 6, pp. 1161-1170, 1991. [http://dx.doi.org/10.1080/00207549108930125]

[14] D. Chang, "Using SLAM to Design the Material Handling System of a Flexible Manufacturing System", Int. J. Prod. Res., vol. 24, no. 1, pp. $15-26,1986$.

[15] T. Warren Liao, "Design of line-type cellular manufacturing systems for minimum operating and material-handling costs", Int. J. Prod. Res., vol. 32, no. 2, pp. 387-397, 1994.

[http://dx.doi.org/10.1080/00207549408956939]

[16] C. Rahul, and W. Subhash, "Impact of Routing Flexibility on the Performance of an FMS-A Simulation Study", Int. J. Flex. Manuf. Syst., vol. 9, pp. 273-298, 1997. [http://dx.doi.org/10.1023/A:1007917429815]

[17] J. Kim I.Roand, "Multi-criteria Operational Control Rules in Flexible Manufacturing Systems", Int. J. Prod. Res., vol. 28, no. 1, pp. 47-63, 1991.

[18] R. Caprihan, and S. Wadhwa, "Impact of routing flexibility on the performance of an FMS-A simulation study", Int. J. Flex. Manuf. Syst., vol. 9, no. 3, pp. 273-298, 1997. [http://dx.doi.org/10.1023/A:1007917429815]

[19] R. Caprihan, A. Kumar, and K.E. Stecke, "A fuzzy dispatching strategy for due date scheduling of FMSs with information delays", Int. J. Flex. Manuf. Syst., vol. 18, no. 1, pp. 29-53, 2006.

[http://dx.doi.org/10.1007/s10696-006-9002-4]

[20] C. Zhao, and W. Zhiming, "A Genetic Algorithm Approach to the Scheduling of FMSs with Multiple Routes", Int. J. Flex. Manuf. Syst., vol. 13, pp. 71-88, 2001. [http://dx.doi.org/10.1023/A:1008148313360]

[21] I.J. Chenand, and C.H. Chung, "Effects of Loading and Routing Decisions on Performance of Flexible Manufacturing Systems", Int. J. Prod. Res., vol. 29, no. 11, pp. 2209-2225, 1991. [http://dx.doi.org/10.1080/00207549108948079]

[22] A.Y. Chang, "On the measurement of routing flexibility: A multiple attribute approach", Int. J. Prod. Econ., vol. 109, pp. 122-136, 2007. [http://dx.doi.org/10.1016/j.ijpe.2006.11.011]

[23] B. Ümit, and F. Murat, "Erinç, A parametric fuzzy logic approach to dynamic part routing under full routing flexibility", Comput. Ind. Eng., vol. 55 , pp. $15-33,2008$.

[http://dx.doi.org/10.1016/j.cie.2007.11.013]

[24] D. Rajamani, N. Singh, and Y.P. Aneja, "Integrated design of cellular manufacturing systems in the presence of alternative process plans", Int. J. Prod. Res., vol. 28, no. 8, pp. 1541-1553, 1990. [http://dx.doi.org/10.1080/00207549008942811]

[25] R. Andrea, and D. Gino, "Flexible job-shop scheduling with routing flexibility and separable setup times using ant colony optimisation method", Rob. Comput-Integr Manuf., vol. 23, pp. 503-516, 2007.

[26] A.C. Garavelli, "Performance analysis of a batch production system with limited flexibility", Int. J. Prod. Econ., vol. 69, pp. 39-48, 2001. [http://dx.doi.org/10.1016/S0925-5273(00)00043-8]

[27] T.Y. ElMekkawy, and H.A. ElMaraghy, "Real-time scheduling with deadlock avoidance in flexible manufacturing systems", Int. J. Adv. Manuf. Technol., vol. 22, pp. 259-270, 2003. [http://dx.doi.org/10.1007/s00170-002-1468-y]

[28] C.S. Shukla, and F.F. Chen, "An intelligent decision support system for part launching in a flexible manufacturing system", Int. J. Adv. Manuf. Technol., vol. 18, pp. 422-433, 2001. [http://dx.doi.org/10.1007/s001700170052]

[29] N.A. Haq, T. Karthikeyan, and M. Dinesh, "Scheduling decisions in FMS using a heuristic approach", Int. J. Adv. Manuf. Technol., vol. 22, no. 5-6, pp. 374-379, 2003.

[http://dx.doi.org/10.1007/s00170-002-1474-0]

[30] F. Mahmoodi, and C.T. Mosier, "The Effects of Scheduling Rules and Routing Flexibility on the Performance of a Random Flexible Manufacturing System", In: International Journal of Flexible Manufacturing Systems, vol. 11. Academic Publishers: Boston, 1999, no. 3, pp. 271-289.

[31] C. Saygin, and S.E. Kilic, "Integrating flexible manufacturing systems with scheduling in flexible manufacturing system", Int. j. adv. Manuf. Technol., vol. 15, no. 4, pp. 268-280, 1999.

[32] L. Ghomri, "Influence des contraintes et des perturbations sur les performances des règles de routages dans un FMS", La conférence 
internationale Conception et Production Intégrées, 2007

[33] A. Hassam, and Z. Sari, "Selection of alternative routings in real time: DMM and modified DMM rules", Int. J. Prod. Dev., vol. 10, no. 1/2/3, pp. 241-258.

[http://dx.doi.org/10.1504/IJPD.2010.029995]

\section{(C) 2017 Houari et al.}

This is an open access article distributed under the terms of the Creative Commons Attribution 4.0 International Public License (CC-BY 4.0), a copy of which is available at: https://creativecommons.org/licenses/by/4.0/legalcode. This license permits unrestricted use, distribution, and reproduction in any medium, provided the original author and source are credited. 